\title{
Ongoing Clinical Trials
}

Section Editor: S. Galandiuk, MD, Louisville

Dig Surg 2007;24:400-408

Published online: August 31, 2007

This section provides information for researchers on clinical trials being in progress in their field throughout the world. The list of trials described herein is by no means inclusive, and the publisher is not responsible for any data given.

Please use the special questionnaire at the end of this section to submit information on a new trial.

The Ongoing Clinical Trials are only available online, free of charge, under www.karger.com/dsu_issues

Oncology

Surgical Infection / Sepsis

Miscellaneous 


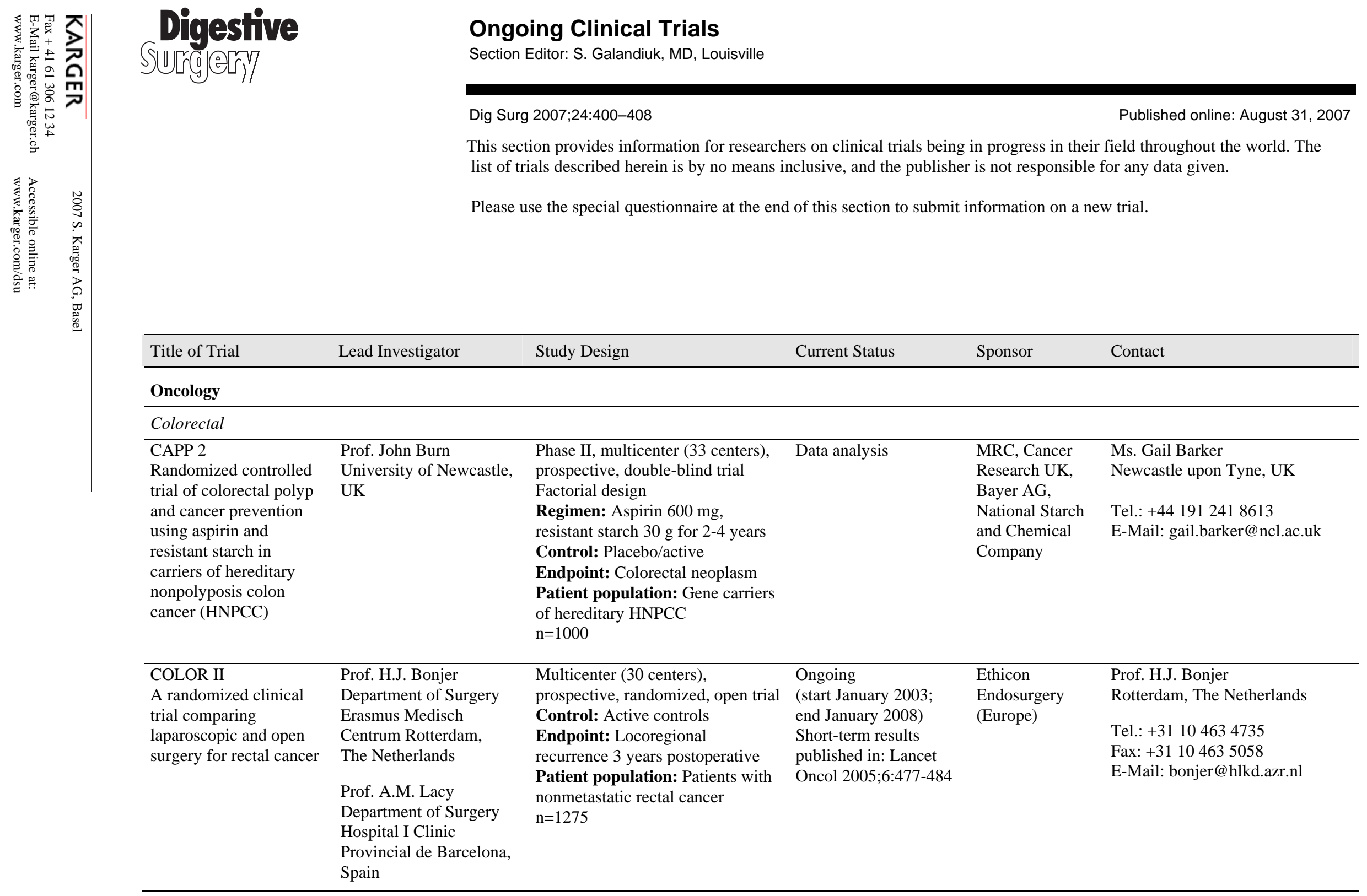


Ongoing Clinical Trials (continued)

\begin{tabular}{lll}
\hline Title of Trial & Lead Investigator & Study Design \\
\hline DaCHS - Prevention of & Prof. Dr. H. Brenner & Independent, case-control trial \\
colorectal carcinoma: & Deutsches Zentrum für & $\mathrm{n}=1500$ \\
The role of screening & $\begin{array}{l}\text { Alternsforschung, } \\
\text { Heidelberg, Germany }\end{array}$ &
\end{tabular}

Current Status

Ongoing (start January

2003; end of recruit-

ment December 2007)

Sponsor

Contact

The role of screening

Heidelberg, Germany

(Deutsches

Dr. Christoph Seiler, MS

Krebsforschungs-

zentrum)

Heidelberg, Germany

Tel.: +496221566986

Fax: +49 6221566988

E-Mail:

christoph_seiler@med.uni-

heidelberg.de

www.sdgc.de

\begin{abstract}
Colon-J-pouch versus
transverse coloplasty

pouch: A randomized

controlled trial

comparing functional

results after rectum

resection and different

reconstructions
\end{abstract}

ISRCTN No.: 78983587

PD Dr. Kaspar Z'graggen Multicenter, prospective,

Klinik Beau-Site, Berne, randomized, open trial

Switzerland

Control: Comparison of different

techniques

Endpoint: Morbidity, function and

mortality during a follow-up period

of 24 months

Patient population: Patients

showing deep rectum pathology

with planned RO-surgery

$\mathrm{n}=150$

Multicenter (12 centers)

prospective, unblinded

(oophorectomy) trial

Control: Patients who do not

undergo oophorectomy

Endpoint: 3-year disease-free

survival

Patient population: Postmeno-

pausal women with colorectal

cancer without evidence of

metastatic disease

$\mathrm{n}=1000$

\section{Additional information:}

Incidence of micrometastases in

ovaria of colorectal cancer patients

and prevention of future

Krukenberg tumors

Dr. Christoph Seiler, MSc

Heidelberg, Germany

Tel.: +49 6221566986

Fax: +49 6221566988

E-Mail:

christoph_seiler@med.uni-

heidelberg.de

www.sdgc.de

Ongoing (start 2003;

end 2007)

Veldhoven, The Netherlands

Tel.: +31408888556

Fax: +31 408888565

E-Mail: r.roumen@mmc.nl 
Ongoing Clinical Trials (continued)

\begin{tabular}{|c|c|c|c|c|c|}
\hline Title of Trial & Lead Investigator & Study Design & Current Status & Sponsor & Contact \\
\hline $\begin{array}{l}\text { Influence of two } \\
\text { different resection } \\
\text { techniques of liver } \\
\text { metastases from } \\
\text { colorectal cancer on } \\
\text { hematogenous tumor } \\
\text { cell dissemination } \\
\text { ISRCTN No.: } 45066244\end{array}$ & $\begin{array}{l}\text { Jürgen Weitz, MD } \\
\text { Department of Surgery, } \\
\text { University of Heidelberg, } \\
\text { Germany }\end{array}$ & $\begin{array}{l}\text { Multicenter ( } 3 \text { centers), } \\
\text { prospective open trial } \\
\text { Control: Comparison of two } \\
\text { techniques } \\
\text { Endpoint: Tumor cell detection in } \\
\text { blood samples } \\
\text { Patient population: Patients with } \\
\text { liver metastases of a colorectal } \\
\text { cancer }\end{array}$ & Ongoing & $\begin{array}{l}\text { Prof. M.W. } \\
\text { Büchler }\end{array}$ & $\begin{array}{l}\text { Dr. Christoph Seiler, MSc } \\
\text { Heidelberg, Germany } \\
\text { Tel.: +496221566986 } \\
\text { Fax: +496221566988 } \\
\text { E-Mail: } \\
\text { christoph_seiler@med.uni- } \\
\text { heidelberg.de } \\
\text { www.sdgc.de }\end{array}$ \\
\hline
\end{tabular}

\section{Pancreatic}

Primary resection vs.

neoadjuvant chemo-

Prof. Dr. W. Hohenberger Multicenter, prospective,

Ongoing (start June

PD Dr. T. Meyer

radiation followed by

University Hospital

nonrandomized trial

2003; 55 patients

Erlangen, Germany

resection for locally

resectable or potentially

resectable pancreatic

Patient population: Patients with

entered to date)

carcinoma without

potentially resectable carcinoma of

Tel.: +49 91318533296

Prof. Dr. G. Grabenbauer the pancreatic head

University Hospital ne254 (127 per arm)

distant metastasis

Erlangen, Germany

Additional information: Pilo

project finished, new trial with gemcitabine and cisplatin as radiosensitizers

Fax: +4991318536595

E-Mail:

thomas.meyer@chir.imed.uni-

erlangen.de

PD Dr. T. Brunner

Erlangen, Germany

E-Mail:

thomas.brunner@strahlen.imed.

uni-erlangen.de

\begin{tabular}{|c|c|c|c|c|c|}
\hline $\begin{array}{l}\text { ESPAC-3(v2) } \\
\text { Adjuvant chemo- } \\
\text { therapies in resectable } \\
\text { pancreatic cancer }\end{array}$ & $\begin{array}{l}\text { Prof. J.P. Neoptolemos } \\
\text { Department of Surgery, } \\
\text { University of Liverpool, } \\
\text { UK }\end{array}$ & $\begin{array}{l}\text { International multicenter (154 } \\
\text { centers) trial; patients with } \\
\text { pancreatic ductal adenocarcinoma } \\
\text { randomized into one of two groups } \\
\text { (gemcitabine; 5-FU and folinic } \\
\text { acid; recruitment closed) and } \\
\text { patients with ampullary or other } \\
\text { tumors of the pancreas randomized } \\
\text { into one of three groups } \\
\text { (gemcitabine; 5-FU and folinic } \\
\text { acid, and observation) } \\
\text { Endpoint: Overall survival } \\
\text { Patient population: Patients with } \\
\text { resected pancreatic cancer } \\
\text { n=1030 }\end{array}$ & Ongoing & $\begin{array}{l}\text { Cancer Research } \\
\text { UK }\end{array}$ & $\begin{array}{l}\text { Mrs. Emily Owen, Coordinator } \\
\text { Liverpool, UK } \\
\text { Tel.: +44 } 1517948932 \\
\text { Fax: +44 } 1517948930 \\
\text { E-Mail: e.owen@liverpool.ac.uk }\end{array}$ \\
\hline
\end{tabular}




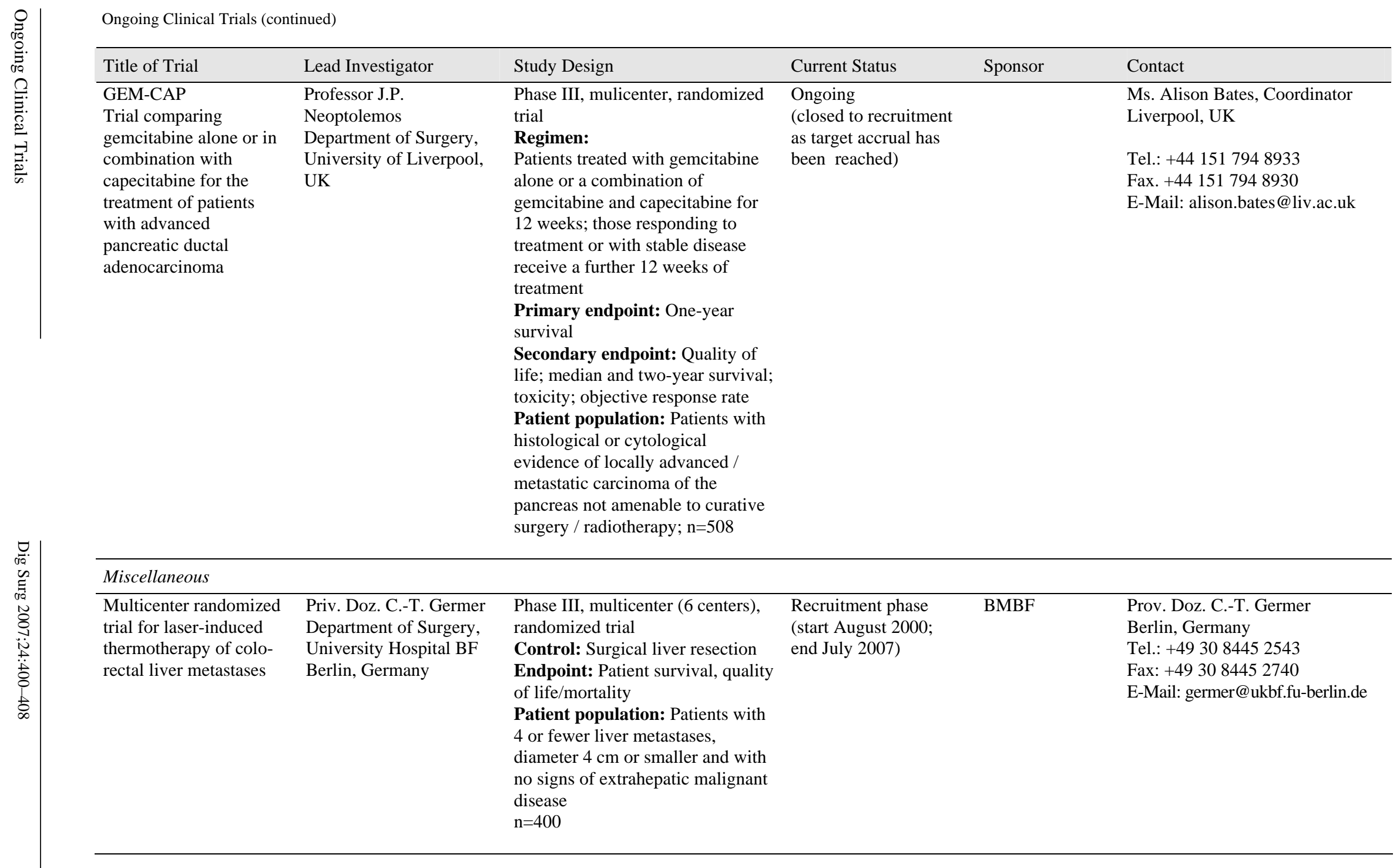


Surgical Infection / Sepsis

Prof. H.G. Gooszen

Multicenter (20 centers of the

Ongoing (start January

The Netherland

Prof. H.G. Gooszen

'step-up approach' vs. Surgery

maximal necrosectomy University Medical

Group), prospective, non-blinded,

2009)

active-controlled trial

in patients with acute

Center Utrecht, The

Patients are randomized between

necrotizing pancreatitis Netherlands

A) Maximal necrosectomy by

Manuscript of design

Health Research

Chairman Dutch Acute

laparotomy with continuous

and rationale of

protocol submitted to

Utrecht, The Netherlands

Pancreatitis

postoperative lavage and B) CT-

BioMed Central,

Tel.: +31302509111

guided percutaneous or endoscopic

transgastric drainage, if necessary

followed by videoscopic assisted

retroperitoneal debridement

(VARD)

Endpoint: Total mortality and

major morbidity

Patient population: Patients with

(suspected) infected necrotizing

pancreatitis, preferably >days after

onset of symptoms

$\mathrm{n}=88$

\begin{tabular}{ll}
\hline PROPATRIA & Prof. H.G. Gooszen \\
Multicenter, & Head, Department of \\
randomized, blinded, & Surgery \\
placebo-controlled trial & University Medical \\
of probiotic prophylaxis & Center Utrecht, The \\
in predicted severe acute & Netherlands \\
pancreatitis & Chairman Dutch Acute \\
& Pancreatitis Study Group
\end{tabular}

Investigator-initiated, multicenter,

double-blind, placebo-controlled

trial

Surgery

egimen: Patients randomly

assigned to receive either live

multispecies probiotics (6 strains,

Ecologica 641) or placebo for 4

Pancreatitis Study Group weeks by nasojejunal tube.

Treatment started within 72 hours

of onset of abdominal pain

Patient Population: Predicted

acute severe pancreatitis

$\begin{array}{lll}\text { Ongoing (start March } & \text { University } & \text { Marc G.H. Besselink, MD } \\ \text { 2004; estimated end } & \text { Medical Center, } & \text { Utrecht, The Netherlands } \\ & \text { Utrecht. } & \\ & \text { Funded by } & \text { Tel.: +31 } 302508074 \\ & \text { Senter, an } & \text { Fax: +31 } 302541944 \\ & \text { agency of the } & \text { E-Mail: } \\ & \text { Ministry of } & \text { m.besselink@umcutrecht.nl } \\ & \text { Economic } & \\ \text { Affairs } & \\ & & \\ & \end{array}$




\section{Miscellaneous}

\section{EUROPAC 2}

Double-blind

randomized controlled

trial to investigate the

efficacy of Antox and

MGCT for the treatment

of hereditary pancreatitis

and idiopathic chronic

pancreatitis

Phase III, double-blind, placebo-

controlled randomized trial

Regimen: Antioxidants for 1 year;

Recruitment

(start 2006)

Magnesium for 1 year

Control: Placebo

Primary endpoint: Reduction in

the number of days of pancreatic

pain

Secondary endpoint: Analgesic

use; hospital admissions; quality of

life scores

Patient population: Patients (5-65

years old) with hereditary pancre-

atitis or ideopathic chronic pancre-

atitis, registered with EUROPAC,

having characteristic pain that is

either intermittent or continuous

\begin{tabular}{|c|c|c|c|c|c|}
\hline $\begin{array}{l}\text { POVATI: Postsurgical } \\
\text { pain outcome in patients } \\
\text { with vertical and trans- } \\
\text { verse abdominal } \\
\text { incision: A randomized } \\
\text { controlled equivalence } \\
\text { trial } \\
\text { ISRCTN Nr.: } 60734227\end{array}$ & $\begin{array}{l}\text { Prof. M.W. Büchler } \\
\text { Department of Surgery } \\
\text { University of Heidelberg } \\
\text { Germany }\end{array}$ & $\begin{array}{l}\text { Single center, prospective, patient- } \\
\text { blind trial } \\
\mathrm{n}=200\end{array}$ & Follow-up & $\begin{array}{l}\text { Prof. M.W. } \\
\text { Büchler }\end{array}$ & $\begin{array}{l}\text { Dr. Christoph Seiler, MSc } \\
\text { Heidelberg, Germany } \\
\text { Tel.: +49 } 6221566986 \\
\text { Fax: +496221566988 } \\
\text { E-Mail: } \\
\text { christoph_seiler@med.uni- } \\
\text { heidelberg.de } \\
\text { www.sdgc.de }\end{array}$ \\
\hline $\begin{array}{l}\text { INSECT: Interrupted or } \\
\text { continuous slowly } \\
\text { absorbable suture } \\
\text { evaluation of abdominal } \\
\text { closure techniques }\end{array}$ & $\begin{array}{l}\text { Prof. M.W. Büchler } \\
\text { Department of Surgery } \\
\text { University of Heidelberg } \\
\text { Germany }\end{array}$ & $\begin{array}{l}\text { Multicenter, prospective, open trial } \\
\text { Control: Comparison of three } \\
\text { techniques } \\
\text { Endpoint: Frequency of } \\
\text { abdominal hernias after } 12 \text { months } \\
\text { and } 3 \text { years } \\
\text { Patient population: Patients who } \\
\text { are planned to undergo an elective } \\
\text { abdominal operation }\end{array}$ & Ongoing & BBD-Aesculap & $\begin{array}{l}\text { Dr. Christoph Seiler, MSc } \\
\text { Heidelberg, Germany } \\
\text { Tel.: +49 } 6221566986 \\
\text { Fax: +496221566988 } \\
\text { E-Mail: } \\
\text { christoph_seiler@med.uni- } \\
\text { heidelberg.de } \\
\text { www.sdgc.de }\end{array}$ \\
\hline
\end{tabular}

Michael G.T. Raraty, MB, BS, PhD, FRCS

EUROPAC Study Coordinator

Liverpool, UK

Tel.: +44 1517064170

Fax: +44 1517065826

E-Mail: mraraty@liv.ac.uk /

europac@liv.ac.uk 
LAPCON: Total laparo- Prof. M.W. Büchler

scopic versus conven- Department of Surgery

tional ileoanal pouch University of Heidelberg

procedure: a randomized Germany

controlled tria
Single center, prospective, open trial

Control: Laparoscopic versus conventional ileoanal pouch

procedure

Endpoint: Intraoperative blood loss

Patient population: Patients with familiar polyposis or ulcerative colitis, scheduled for elective proctocolectomy with ileoanal pouch
Ongoing

Büchler

Dr. Christoph Seiler, MS

Heidelberg, Germany

Tel.: +496221566986 Fax: +49 6221566988

E-Mail:

christoph_seiler@med.uniheidelberg.de

www.sdgc.de

\begin{tabular}{ll}
\hline CLIVIT: Clips vs. & Prof. M.W. Büchler \\
ligatures. A multicenter & Department of Surgery \\
randomized controlled & University of Heidelberg \\
trial & Germany
\end{tabular}

trial

Multicenter, prospective, open trial Ongoing

Control: Comparison of two

techniques to control vessels in

thyroid surgery

Endpoint: Time of resection

Patient population: Patients who

are planned to undergo an elective

bilateral thyroid resection

Dr. Christoph Seiler, MS Heidelberg, Germany

Tel.: +49 6221566986 Fax: +49 6221566988

E-Mail:

christoph_seiler@med.uni-

heidelberg.de www.sdgc.de

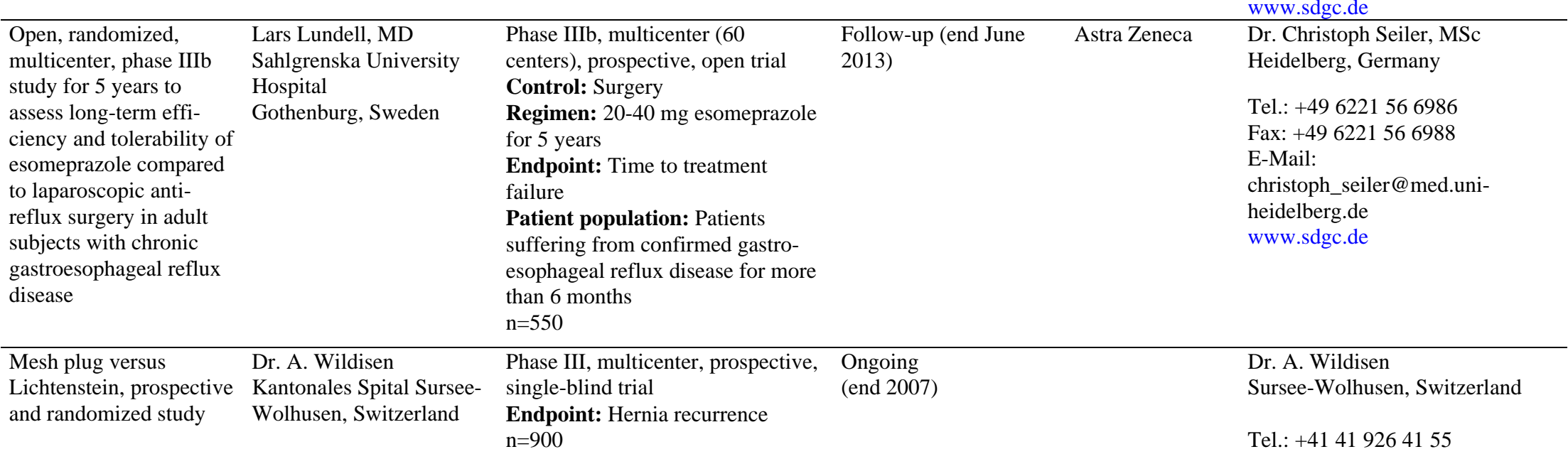


Ongoing Clinical Trials (continued)

A phase III, double-

blind, randomized,

parallel-group, placebo-

controlled study of

intravenous (IV)

methylnaltrexone

bromide (MNTX) in the

treatment of post-

operative ileus (POI)

Phase III, multicenter trial (75-90 Ongoing

centers worldwide)

Control: Placebo

Regimen: IV MNTX $24 \mathrm{mg}$,

MNTX $12 \mathrm{mg}$, or placebo for up to

10 days

Endpoint: Length of time from

end of surgery to first bowel

movement

Patient Population: Patients

scheduled for a segmental

colectomy via open laparotomy

with general anesthesi
Progenics

Pharmaceutical, Louisville, Ky., USA

Inc.

Tel.: +1 5025830880

Fax: +1 5025852988

E-Mail:

aowill01@gwise.louisville.edu 
Digestive

surgergery

\section{Questionnaire}

\section{for Trial Submission}

To submit information on a clinical trial for publication in the 'Ongoing Clinical Trials' section, please complete this short questionnaire.

\section{1) Title of study (in full)}

\section{2) Lead investigator}

Title:

Name:

Affiliation:

\section{3) Field of study}

Please indicate one or more of the following categories:

$\square$ Oncology $\square$ Inflammatory Bowel Disease

$\square$ Motility $\square$ Surgical Infection/Sepsis

$\square$ Miscellaneous

Other (please specify):

\section{4) Study phase (drug trials only)}

Please indicate one of the following categories:

$\square$ Phase I $\square$ Phase II $\square$ Phase III $\square$ Phase IV

$\square$ Independent (investigator initiated)

\section{5) Study design}

$\square$ Multicenter $\square$ Single center (please indicate as appropriate) If multicenter, please state how many centers are involved:

$\square$ Prospective $\square$ Retrospective (please indicate as appropriate)

If prospective, level of randomization (e.g. single blind, double blind, investigator-blind):

Controls (i.e. placebo-, active-, etc.):

Cross-over design (one-way, two-way, etc.):

Duration of therapy (if applicable):

\section{KARGER}

Dosing regimen (if applicable):

Primary study endpoint:

Other information on study design (please include any other details that might be relevant):

\section{6) Patients}

Number of patients (planned):

Patient population (e.g. patients with mild UC previously controlled on...):

\section{7) Current status of study}

Start date:

Estimated end date:

Status (please indicate as appropriate):

$\begin{array}{ll}\square \text { Development } & \square \text { Recruitment } \quad \square \text { Ongoing } \\ \square \text { LPO } & \square \text { Analysis in progress }\end{array}$

$\square$ LPO $\quad \square$ Analysis in progress

\section{Publication}

Abstract submitted to:

Manuscript submitted to:

\section{8) Sponsor}

\section{9) Contact for further information:}

Name:

Address:

Telephone:

Fax:

E-Mail:

Please return the completed questionnaire as soon as possible to the Section Editor:

Susan Galandiuk, MD

Department of Surgery

School of Medicine

University of Louisville

Louisville, KY 40292, USA

Tel.: +1 5028525442

Fax: +1 5028528915 or contact:

S. Karger AG

Attn.: Ms. Yvonne Rebmann

Allschwilerstrasse 10

PO Box

CH-4009 Basel

Tel.: +41613061351

Fax: +41613061234

E-Mail: y.rebmann@karger.ch 